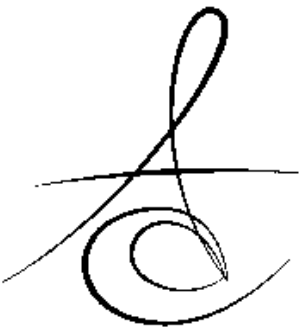

\title{
FARKLI PREPARASYON TEKNİKLERİ VE FARKLI SİMAN ARALIKLARINDA HAZIRLANAN CAD/CAM LAMİNATE VENEERLERİN KIRILMA DAYANIMLARININ DEĞERLENDİRİLMESİ
}

\section{FRACTURE STRENGTH EVALUATION OF CAD/CAM LAMINATE VENEERS PREPARED WITH DIFFERENT METHODS AND DIFFERENT CEMENT THICKNESSES}

\author{
Uzm. Dt. Orhan Rıdvan VURGEÇ* Uzm. Dt. Özcan KARATAS* \\ Yrd. Doç. Dr. Ömer SAĞSÖZ* \\ Prof. Dr. Nilgün SEVEN*
}

Makale Kodu/Article code: 2739

Makale Gönderilme tarihi: 29.03.2016

Kabul Tarihi: 22.04.2016

\section{ÖZET}

Amaç: Bu çalışmanın amacl; 2 farklı siman aralığında ve 2 farklı preparasyon tekniği ile CAD/CAM kullanılarak hazırlanan laminate veneerlerin kırılma direncini karşılaştırmaktadır.

Gereç ve Yöntem: Çalışmada 40 adet benzer boyutlarda seçilen üst anterior diş kullanıldı. Her dişe laminate preparasyonu yapıldıktan sonra laminate veneer ile restore edildi. Dişler, preparasyon teknikleri; insizalde sonlanan geleneksel kesim ve insizali içine alan kesim ile siman aralıkları; $50 \mu \mathrm{m}$ ve $200 \mu \mathrm{m}$ olacak şekilde 4 gruba ayrıldı. Laminateler feldspatik seramik bloklar kullanılarak Cerec CAD/CAM cihazı yardımıyla hazırlanıp self etch rezin siman ile uygulandı. 125 derecelik açıyla kendinden sertleşen akril silindirik bloklara gömülen dişlere üniversal test cihazıyla (Instron 3344) sabit hızda kuvvet uygulanarak kırma testi gerçekleştirildi. Laminatelerin kırılma kuvvetleri kaydedildi. Elde edilen sonuçlar SPSS 20 istatistik yazılımı kullanılarak tek yönlü varyans analizi ve Tukey post hoc testi kullanılarak istatiksel olarak değerlendirildi.

Bulgular: Siman aralığı karşılaştırıldığında 50 m, 200 um siman aralığına göre daha yüksek kırılma direnci gösterdi. Kesim teknikleri karşılaştırıldığında geleneksel kesim insizale overlap kesime göre daha yüksek kırılma direnci gösterdi. En yüksek ortalama kırılma direnci geleneksel kesim ve $50 \mu \mathrm{m}$ siman aralığında (643 N), en düşük değer insizale overlap kesim ve $200 \mu \mathrm{m}$ siman aralığında (359.8 N) kaydedildi.

Sonuç: Geleneksek kesim tekniği laminate veneerlerin kırılma direncini arttırır. Siman aralığı azaldıkça kırılma direnci artar.

Anahtar kelimeler: Kesim tekniği, kırılma direnci, laminate veneer, siman aralığı.

\section{ABSTRACT}

Aim: The aim of this investigation is to evaluate fracture resistance of veneer materials which prepared in two different methods with two different cement thicknesses by CAD/CAM.

Material and Method: Forty maxillary incisors in similar sizes were used in this study. Each tooth restored with laminate after preparation. Teeth were divided into 4 groups according to preparation techniques; traditional preparation and incisal-overlap preparation, and cement thicknesses; $50 \mu \mathrm{m}$ and 200 $\mu \mathrm{m}$. Laminates were milled with Cerec CAD/CAM device (Sirona) from feldspathic ceramic blocks and applied using self etch resin cement. Fracture tests were performed applying load with constant speed using a universal testing machine (Instron 3344) to teeth embedded into cylindrical self-cure acrylic resin blocks with 125-degree angle. Fracture load values of laminates were recorded. Results were evaluated statistically with one-way ANOVA and Tukey post hoc tests using SPSS 20 software.

Results: In terms of cement thickness, $50 \mu \mathrm{m}$ showed statistically higher fracture strength values than $200 \mu \mathrm{m}$. In terms of preparation technique, traditional technique showed higher fracture strength than incisal-overlap technique. The highest mean fracture strength was recorded for traditional preparation with $50 \mu \mathrm{m}(643 \mathrm{~N})$, while the lowest was for incisal-overlap preparation with $200 \mu \mathrm{m}$ (359.8 N).

Conclusion: Traditional preparation technique increases fracture strength of laminate veneers. Fracture strength increases as cement thickness reduced.

Keywords: Preparation technique, fracture resistance, laminate veneer, cement thickness

*Atatürk Üniversitesi, Diş Hekimliği Fakültesi, Restoratif Diş Tedavisi Anabilim Dalı 


\section{GİRİ}

Dental seramikler diş eksikliği veya bozuk diş yapılarını düzeltme amacı ile tasarlanmış sistemlerin parçası olan malzemelerdir. ${ }^{1}$ Seramik materyallerin üretim metotları ve mekanik özelliklerinde son yıllarda önemli ilerlemeler kaydedilmiştir. ${ }^{2}$ Günümüzde sosyoekonomik durumun gelişmesi ile birlikte estetik ihtiyaç artmaktadır. Laminate veneerler bu estetik intiyacın karşılanmasında önemli bir yer tutmaktadır. Seramik laminate veneer kullanımı ile restorasyonlarda efektif ve minimal invaziv bir yaklaşım ortaya çıkmıştır. Seramik materyallerdeki gelişmeler, adeziv teknoloji ve klinik teknikler seramik laminate veneerlerin minimal estetik diş hekimliği alanında bir tedavi seçeneği olmasını sağlamıştır. ${ }^{3,4}$

Bilgisayar ve teknolojideki ilerlemeler sayesinde, son yıllarda artık CAD/CAM yardımıyla üretilen restorasyonlarda hep aynı kalite standardı yakalanabilmekte ve hastaya uygun başarılı restorasyonlar hazırlanabilmektedir. 5, 6

Laminate veneerlerde seramiğin tek başına kullanımı literatürde kırılma direnci açısından tartışma konusu olmuştur. Bütün dental seramikler diğer dental materyallerle karşılaştırıldığında düşük kırılma dayanımı gösterirler. ${ }^{7}$ Bununla birlikte seramik yapısındaki güçlendirme çalışmaları tam seramik restorasyonların yapımını mümkün kılmaktadır. Yine de dental seramiklerin oral kavitedeki fonksiyonel kuvvetlere karşı dayanımlarındaki yetersizlik önemli bir dezavantajlarıdır. Seramik veneerlerin en sık klinik başarısızlığı kırılma veya bağlantının bozulması sonucu oluşur. Bunlar içerisinde kırıma tüm başarısızlıkların içinde \%67'lik önemli bir oranla en büyük paya sahiptir.8,9

Seramik veneerlerin 15 yıllık gözlem sonrası başarı oranları \%93 olarak rapor edilmiştir. Bu başarı kriterleri diş yapısının korunması, mineye bağlanma, iyi estetik ve renk uyumunu içermektedir. ${ }^{10}$ Laminate restorasyonlarda preparasyon dizaynı en çok tartışılan konulardan biri olmaya devam etmektedir. İdeal diş preparasyonu esnasında tedavi planlaması, uygun fonksiyon ve estetik kritik bir önem arz etmektedir. ${ }^{11,12}$ Genel olarak labial yüzde sınırlı kalan pencere kesim, insizal kenara ulaşan kesim ve insizal kenarı içine alıp palatinale uzanan kesim şeklinde preparasyon teknikleri vardır. ${ }^{13,14}$ İnsizal kenar bütünlüğünü bozmadan uygulanan kesim tekniği dişin kesici kenarı kısaltılmadan fasial yüzde yapılan aşındırmadır, insizal kenarda sonlandırılır. İnsizal kenarı içerisine alan teknikte ise kesici kenar $2 \mathrm{~mm}$ kısaltılarak dişin palatinali 1,5-2 mm kadar kesime dahil edilir. Bu preparasyon dizaynı bağlantı yüzeyini arttırır ve laminate veneerlerin simantasyon esnasında doğru yerleşmesine rehberlik yapar. ${ }^{15,} 16$ İnsizal kenarda sonlandırılan kesim tekniğinin insizal kenarı içine alıp palatinale uzanan kesim tekniğine göre daha az çekme streslerine maruz kaldığı öne sürülmüştür. ${ }^{17,}{ }^{18}$ Buna karşın 3 boyutlu sonlu elemanlar analizi çalışmalarında palatinale uzanan preparasyonda daha iyi stres dağılımının gözlendiği belirlenmiştir. ${ }^{19}, 20$

Wilson ve arkadaşlarının ${ }^{21}$ metal güdük üzerinde yaptıkları bir çalışmada, siman aralığının artışı ile seramik restorasyonlarda kırılma dayanımının azaldığı gösterilmiştir. Son zamanlarda laminate veneerlerin preparasyon tekniklerinin kırılma dayanımı üzerine olan etkisi birçok araştırmada rapor edilmiştir. ${ }^{7-9}$ Ancak farklı siman aralıklarının laminate kırıma dayanımı üzerindeki etkisi bilinmemektedir. $\mathrm{Bu}$ nedenle bu çalışmada farklı siman aralıklarında farklı kesim teknikleri ile hazırlanan seramik laminatelerin kırılma dayanımlarının in-vitro olarak incelenmesi amaçlanmıştır. Çalışmamızın sıfır hipotezi farklı siman aralıklarının ve farklı kesim tekniklerinin seramik laminatelerin kırıma dirençlerine etki etmeyeceği şeklinde belirlenmiştir.

\section{GEREÇ-YÖNTEM}

Çalışmada 40 adet çekilmiş, çürüksüz, 10 mm kron boyuna ve $8 \mathrm{~mm}$ mesio-distal genişliğe sahip benzer boyutlardaki hipoplastik defekt ve çatlak bulunmayan insan üst santral keser diş kullanıldı. (Karar no: 23/2015, Atatürük Üniversitesi Diş Hekimliği Fakültesi Etik Kurulu, Erzurum, Türkiye) Dişin kesici kenarı kısaltılmadan fasiyal yüzde aşındırma yapılarak preparasyon dişin kesici kenarında sonlandırılan geleneksel veneer ve dişin kesici kenarını içersine alacak şekilde sonlandırılan insizal overlap olmak üzere laminate preparasyonu için iki farklı kesim tekniği kullanıldı. (İnsizal overlap ve geleneksel veneer Preparasyonu tamamlanan dişler iki farklı siman aralığıyla restore edilecek şekilde 4 gruba ayrıldı. $(n=10)$ Grup 1; geleneksel kesim tekniği $+50 \mu \mathrm{m}$ siman aralığı, Grup 2; geleneksel kesim tekniği + $200 \mu \mathrm{m}$ siman aralığı, Grup 3; insizale overlap tekniği + $50 \mu \mathrm{m}$ siman aralığı, Grup 4; insizale overlap tekniği + $200 \mu \mathrm{m}$ siman aralığı. 
Diş kesimleri su soğutması altında aeretör ile yapıldı. Geleneksel veneer preparasyonunda dişlerin facial yüzü kolede $0,3 \mathrm{~mm}$, orta insizal üçlüde $0.5 \mathrm{~mm}$ kalınlığında ve sementoenamel birleşim seviyesinde kesildi. İnsizal overlap preparasyonunda dişlerin insizal kenarları $2 \mathrm{~mm}$ kalınlığında ve fasiyal yüz traditional preparasyonla aynı şekilde kesildi. Preparasyonlar bitirme frezi ile düzeltildi. Preparasyonu yapılan dişlerin yüzeylerine yansıtıcı sprey sıkılarak bir ağız içi tarayıcı (Cerec BlueCam, Sirona) ile optik ölçüleri alındı. Alınan ölçülere göre $50 \mu \mathrm{m}$ ve $200 \mu \mathrm{m}$ siman aralığında feldspatik seramiklerden (Cerec Blocs, Sirona, lot no:37810) laminate restorasyonlar hazırlandı. Siman aralıkları ve laminate kalınlıkları CEREC 4 bilgisayar yazııımı ile oluşturuldu. Laminateler CEREC MCXL freze cihazı ile hazırlanarak bir self adeziv rezin siman (Rely X U200, 3M ESPE, lot no:515011) ile parmak baskıSI altında yapıştıııldı. Fazla siman restorasyon kenarlarından uzaklaştırılarak $1 \mathrm{dk}$ süreyle bir LED polimerizasyon cihazıyla (Elipar S10, 3M ESPE) polimerizasyonu yapıldı. Örnekler 1 hafta süre ile $37^{\circ} \mathrm{C}$ distile suda bekletildi. Tüm örnekler $125 C^{\prime}$ 'ik açılla silindirik şekilli akrilik bloklara gömüldü. Kırılma direnci testi, örneklerin insizal kenarları üzerine polietilenden yapılmış stres emici bir disk yerleştirilerek, $3 \mathrm{~mm}$ çapında kırııı uç vasıtasıyla Instron 3344 universal test cihazı ile $1 \mathrm{~mm} / \mathrm{dk}$ hızda gerçekleştirildi. Newton (N) olarak kaydedilen veriler tek yönlü varyans analizi ve Tukey post-hoc testi ile SPSS 20 (IBM Statistics) yaziımı kullanılarak analiz edildi. $(a=0.05)$

\section{BULGULAR}

Bu çalışmada elde edilen verilere göre, gruplar arasında istatistiksel olarak anlamlı bir fark bulundu. $(p<0.05)($ Tablo- 1$) \quad$ Teknikler karşılaştırıııı̆ında Grup 1 diğer gruplara göre daha yüksek kırıma direnci göstermiştir. Kesim tekniklerine göre karşılaştırıldıklarında $50 \quad \mu \mathrm{m}$ siman aralığında hazırlanan laminatelerde geleneksel kesim yapılan grup, insizale overlap yapılan gruptan daha yüksek kırılma direnci gösterirken $(p<0.05) ; 200 \mu \mathrm{m}$ siman aralığında hazırlanan laminatelerde geleneksel kesim yapılan grup ile insizal overlap kesim yapılan grup arasında istatistiksel olarak anlamlı bir fark bulunamamışır ( $p>0.05$ ).

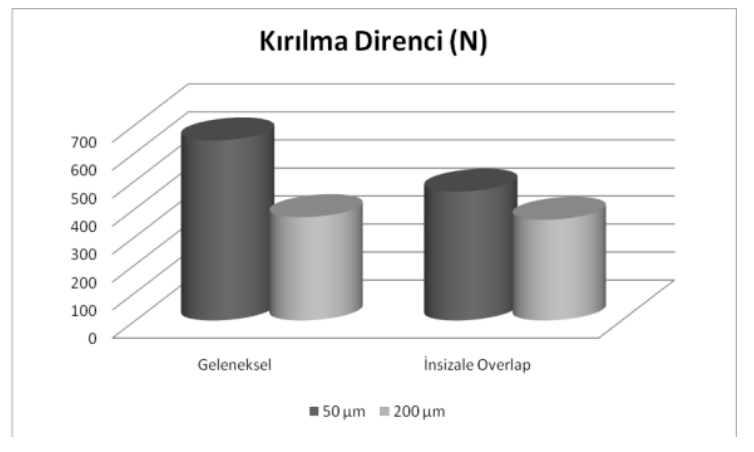

Siman aralıklarına göre karşılaştııılıklarında ise geleneksel kesim yapılan gruplar içerisinde $50 \mu \mathrm{m}$ siman aralığında hazırlanan laminateler $200 \mu \mathrm{m}$ siman aralığında hazırlanan laminatelere göre daha yüksek kırıma direnci göstermiştir; insizale overlap kesim yapılan gruplar içerisinde $50 \mu \mathrm{m}$ siman aralığında hazırlanan laminateler $200 \mu \mathrm{m}$ siman aralığında hazırlanan laminatelere göre daha yüksek kırıma direnci göstermiştir.

\begin{tabular}{|c|c|c|}
\hline \multirow{2}{*}{ Kesim Tekniği } & $\begin{array}{c}\text { Siman } \\
\text { Aralığı }\end{array}$ & Ortalama + SD \\
\hline \multirow{2}{*}{ Geleneksel } & $50 \mu \mathrm{m}$ & $643,01 \pm 36,20^{\mathrm{A}}$ \\
\cline { 2 - 3 } & $200 \mu \mathrm{m}$ & $369,08 \pm 27,74^{\mathrm{B}}$ \\
\hline İnsizale Overlap & $50 \mu \mathrm{m}$ & $460,31 \pm 25,11^{\mathrm{C}}$ \\
\cline { 2 - 3 } & $200 \mu \mathrm{m}$ & $359,81 \pm 26,74^{\mathrm{B}}$ \\
\hline
\end{tabular}

*Aynı harflerle gösterilen değerler arasında istatiksel olarak anlamlı farklılık yoktur.

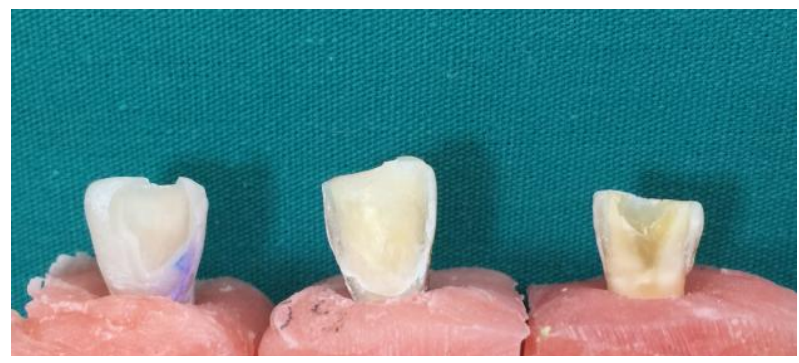

A)Koheziv kırılma

B) Miks Kırılma

C)Adeziv kırılma

Resim 1. (Kırılma Şekilleri)

\section{TARTIŞMA}

Dişlerin dehidratasyonunu önlemek için, çekilmiş dişlerin deneyler yapılıncaya kadar bekletileceği solüsyonlar $\% 10$ formol, $\% 0,1$ timol ve distile su olarak kullanılabilmektedir. ${ }^{22-25}$

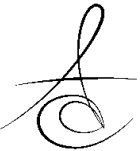


Kırılma direnci, çekme, makaslama ve mikrosızıntı testleri in vitro ortam testlerin en çok kulanılanlarıdır. ${ }^{26,}{ }^{27}$ Van Meerbeek ve arkadaşlarının ${ }^{28}$ yaptıkları çalışmaya göre, laminate veneerlerde makaslama bağlanma dayanımı ile restorasyon-diş arası bağlanma kuvveti ölçülürken, materyalin dayanıklılığı kırılma direnci kuvvet dayanımı testiyle ölçülmektedir.

Lin ve arkadaşlarının ${ }^{29}$ yaptığı bir çalışmada, kırıcı ucun $125^{\circ}$ açıyla kesici kenardan uygulanmasının, ağız içinde laminate veneerlerin maruz kaldığı kuvvetin yönünü daha iyi taklit ettiği gösterilmiştir, bu çalışmada literatür araştırması sonucu elde ettiğimiz veriler ışığında kırıcı ucu $125^{\circ}$ açıyla kesici kenardan uygulandı.

Veneer restorasyonlar için yapılabilecek kesimler, McLaughlin ${ }^{30}$ tarafından 6 ana dizayn olarak bildirilmiştir. Tanımlanan kesimlerin en çok kullanılanları; insizale overlap, feather edge, bevel ve mine içi pencere tipi kesimlerdir. Birçok araştırmacı tarafından, palatinal kenarın bir kısmını içeren, insizal kenarda doğal dişlerin daha iyi taklit edilmesini sağlayabilerek estetik olarak tatmin edici olan ve insizal kenarın oklüzal kuvvetlere direncini arttıran, insizal overlap kesim tekniği önerilmektedir. ${ }^{31,} 32$

Highton ve arkadaşları ${ }^{33}$, 34 iki boyutlu foto elastik stres analizi yaptıkları çalışmalarında, insizal overlap preparasyonun oklüzal kuvveti geniş alana dağıtarak laminate veneerde meydana gelecek stres yoğunlaş- masını azalttığını göstermişlerdir.

May ve arkadasları ${ }^{35}$ çalışmalarında hem in-vitro hem de sonlu elemanlar stres analizi bulgularına göre siman kalınlığının artmasıyla kırıma direncinin düştüğünü göstermişlerdir. Sonlu elemanlar analizi yapılırken siman kalınlığı tam olarak belirlenir. Bununla birlikte in-vitro çalışmalarda hazırlanılması istenilen siman kalınlığı tam olarak ayarlanamaz ve yakın değerler elde edilir. ${ }^{36-38}$

Meijering ve arkadaşlarıysa ${ }^{39}$ 2,5 yıllık klinik takip yapılan bir çalışmada, insizal kenarın preparasyon şeklinin restorasyonun başarısı ile ilişkili olmadığını göstermişlerdir. Başka bir çalışmada veneer kalınlıklarında farklılık olsa da, zirkonya veneerlerin basma kuvveti başarısızlığında preparasyon dizaynından kaynaklanan bir etki bulunmamıştır. ${ }^{40}$ Lin ve arkadaşları ${ }^{29}$ da geleneksel kesim ve insizale overlap kesim arasında kırılma dirençleri arasında belirli bir farkılık bulamamışlardır. Bu çalışmada ise insizale overlap veneer kesim tekniğinde, geleneksel veneer kesim tekniğine göre daha düşük kuvvette kırıma dayanımı ölçülmüştür. Geleneksel kesimde diş yapısında daha fazla mine kaldığından kırılma direncinin artması söz konusudur. ${ }^{40}$

Troedson ve arkadaşları ${ }^{41}$, preparasyonun şeklinden çok dişe gelen çiğneme kuvvetinin yönünün restorasyonun başarısı için önemli olduğunu bildirmişlerdir.Restorasyonun başarısı için önemli olan bir diğer etkense mine miktarı ve kalitesidir. Preparas- yonun en az \%50'sinin mine üzerinde olmasının klinik başarıyı önemli ölçüde arttırdığı daha önceki çalışma- larda gözlenmiştir. ${ }^{3,42}$

Uniform olmayan siman kalınlığı simanın bağlanma gücünü aşan değerlerde maksimum makaslama stresini dayanım gücünü azaltabilir. Bu nedenle siman tabakası mümkün olduğunca ince ve uniform olmalıdır. ${ }^{43} \mathrm{Bu}$ çalışmada CAD yazılımı üzerinde istenilen siman aralıkları belirlenmiş ve uniform olarak hazılanabilmiştir.

$\mathrm{Bu}$ çalışmada, $50 \mu$ siman aralığında oluşan ortalama kırılma kuvvetinin $200 \mu$ siman aralığından önemli ölçüde yüksek olması $(p<0.05)$ siman aralığı arttıkça kırılmaya direncin azalması şeklinde bir sonuç ortaya çıkarmıştır. Tuntiprawan ve Wilson ${ }^{21}$, farklı siman aralığında porselen jaket kuronların kırılma dirençleri arasında benzer bir ters orantının olduğunu bildirmişlerdir. Bu durumu yük altında simanın şekil değiştirmesine ve siman aralığının nispeten fazla olduğu gruplarda porselenin simanın içerisine doğru daha fazla deforme olmasına ve daha düşük kuvvet altında ve daha erken kırılmasına bağlamışlardır. Siman aralığının nispeten az olduğu gruplarda ise, porselen alttaki güdüğe kırılmadan hemen yaklaşacak ve sıkıştırma kuvvetlerine çekme kuvvetlerine göre daha fazla dayanıklı olduğu için daha yüksek değerlerde kırılacaktır. Benzer şekilde Scherrer ve arkadaşları $^{44}$ da rezin siman kullanarak cam seramiklerin kırılma direncini incelemişler ve $26 \mu$ ile $297 \mu$ siman aralıkları arasında istatistiksel olarak anlamlı bir fark bulmuşlardır. Pirakki ve arkadaşları ${ }^{45}$ ise, $1 \mathrm{~mm}$ kalınlığındaki seramiğin kırılma direncinin siman aralığı arttıkça arttığını bulmuşlardır. $2 \mathrm{~mm}$ kalınlığında seramik kullandıklarında ise farklı siman aralığı değerlerinin kırılma direnci üzerinde etkisinin olmadığını ileri sürmüşlerdir. Farklı çalışmalarda kullanılan farklı örnek boyutu, seramik ve simanlar kırılma direnci üzerinde etkili olabilir. ${ }^{46}$ Mevcut çalışmamızda da laminate kırılma direnci test edildiği

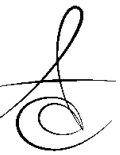


için labialde seramik kalınlığı $0.3 \mathrm{~mm}$ olarak ayarlandı. $\mathrm{Bu}$, siman aralığındaki boyut değişimlerinin kırılma direnci üzerindeki etkisini ön plana çıkarabilecek bir durumdur.

Rezin simanın polimerizasyon büzülmesi esnasında oluşacak gerilmeler siman ile seramik arasında bağlantının bozulmasına neden olabilir. Artan siman aralığında daha fazla büzülme ve bağlantıda bozulma meydana gelir. Yapılan birçok çalışmada simante edilmemiş restorasyonların simante edilen restorasyonlara göre daha düşük kırıma direnci gösterdiği bildirilmiştir. ${ }^{45-47} \mathrm{Bu}$ nedenle artan siman aralığı ve polimerizasyon büzülmesi ile bağlantı zayıflayacağından veya bazı mikro-bölgelerde bağlantı kopacağından kırıma direnci de azalıyor olabilir.

\section{SONUÇLAR} bulunmuştur;

Bu çalışmaya dayanarak aşağıdaki sonuçlar

- Daha konservatif olan geleneksel kesim tekniği kırıma direnci açısından insizale uzanan kesim tekniğine göre daha başarılıdır. Kalan diş dokusu miktarının fazla olması laminate veneerlerde başarıyı arttırır.

-Preparasyon tekniği değişmeksizin $50 \mu$ ve $200 \mu$ Siman aralıkları arasında kırıma kırıma direnci açısından farklılık gözlendi. Sonuç olarak siman aralığı arttıkça kırıma direnci düşer.

\section{KAYNAKLAR}

1. Rosenblum MA, Schulman A. A review of allceramic restorations. The Journal of the American Dental Association, 1997, 128: 297-307.

2. Shenoy A, Shenoy N. Dental ceramics: An update. Journal of conservative dentistry, 2010, 13: 195.

3. Peumans $M$, Van Meerbeek $B$, Lambrechts $P_{r}$ Vanherle G. Porcelain veneers: a review of the literature. Journal of dentistry, 2000, 28: 163-77.

4. Kelleher MG DS, Lewis N. Ethical marketing in 'aesthetic' ('esthetic') or 'cosmetic dentistry' part 2. Dent Update, 2012: 39: 390-2, 394-6, 398-400 passim. Baskı.

5. KARAALIOĞLU AGDOF, DUYMUŞ ZY. Diş hekimliğinde uygulanan CAD/CAM sistemleri. Atatürk Üniv Diş Hek Fak Derg 2008; 18.
6. Nakamura M, Matsumura $\mathrm{H}$. The 24-year clinical performance of porcelain laminate veneer restorations bonded with a two-liquid silane primer and a tri-n-butylborane-initiated adhesive resin. Journal of oral science, 2014, 56: 227-30.

7. Rizkalla $A S$, Jones DW. Indentation fracture toughness and dynamic elastic moduli for commercial feldspathic dental porcelain materials. Dental Materials, 2004, 20: 198-206.

8. Friedman M. A 15-year review of porcelain veneer failure--a clinician's observations. Compendium of continuing education in dentistry (Jamesburg, NJ: 1995), 1998, 19: 625-8, 630, 2 passim; quiz 638.

9. BARGHI N, BERRY TG. Post-bonding Crack Formation in Porcelain Veneers. Journal of Esthetic and Restorative Dentistry, 1997, 9: 51-4.

10. Edelhoff $D$, Sorensen JA. Tooth structure removal associated with various preparation designs for anterior teeth. The Journal of Prosthetic Dentistry, 2002, 87: 503-9.

11. Magne P, Kwon K-R, Belser UC, Hodges JS, Douglas WH. Crack propensity of porcelain laminate veneers: a simulated operatory evaluation. The Journal of Prosthetic Dentistry, 1999, 81: 327-34.

12. Stevenson B, Ibbetson R. The effect of the substructure on the colour of samples/restorations veneered with ceramic: a literature review. Journal of dentistry, 2010, 38: 361-8.

13. Stappert CF, Ozden U, Gerds T, Strub JR. Longevity and failure load of ceramic veneers with different preparation designs after exposure to masticatory simulation. The Journal of Prosthetic Dentistry, 2005, 94: 132-9.

14. Magne P, Douglas WH. Design optimization and evolution of bonded ceramics for the anterior dentition: a finite-element analysis. Quintessence international, 1999, 30.

15. Gürel G. Predictable, precise, and repeatable tooth preparation for porcelain laminate veneers. Practical procedures $\&$ aesthetic dentistry: PPAD, 2002, 15: 17-24; quiz 26.

16. Garber D. Rational tooth preparation for porcelain laminate veneers. Compendium (Newtown, Pa.), 1991, 12: 316, 318, 20 passim.

17. Castelnuovo J, Tjan AH, Phillips K, Nicholls JI, Kois JC, of Washington U, of Dentistry S. Fracture load and mode of failure of ceramic veneers with 
different preparations. The Journal of Prosthetic Dentistry, 2000, 83: 171-80.

18. Magne $\mathrm{P}$, Douglas $\mathrm{WH}$. Porcelain veneers: dentin bonding optimization and biomimetic recovery of the crown. International Journal of Prosthodontics, 1999, 12.

19. Zarone F, Apicella D, Sorrentino R, Ferro V, Aversa $\mathrm{R}$, Apicella $A$. Influence of tooth preparation design on the stress distribution in maxillary central incisors restored by means of alumina porcelain veneers: a 3D-finite element analysis. Dental Materials, 2005, 21: 1178-88.

20. Zarone F, Epifania E, Leone G, Sorrentino R, Ferrari $M$. Dynamometric assessment of the mechanical resistance of porcelain veneers related to tooth preparation: a comparison between two techniques. The Journal of Prosthetic Dentistry, 2006, 95: 354-63.

21. Tuntiprawon M, Wilson PR. The effect of cement thickness on the fracture strength of all-ceramic crowns. Australian dental journal, 1995, 40: 17-21.

22. Schittly E, Le Goff S, Besnault C, Sadoun M, Ruse $N$. Effect of water storage on the flexural strength of four self-etching adhesive resin cements and on the dentin-titanium shear bond strength mediated by them. Operative dentistry, 2014, 39: E171-7.

23. Keul C, Liebermann A, Roos M, Uhrenbacher J, Stawarczyk B. The effect of ceramic primer on shear bond strength of resin composite cement to zirconia: a function of water storage and thermal cycling. The Journal of the American Dental Association, 2013, 144: 1261-71.

24. Konno ANK, Sinhoreti MAC, Consani S, Sobrinho LC, Consani RLX. Storage effect on the shear bond strength of adhesive systems. Brazilian dental journal, 2003, 14: 42-7.

25. Titley K, Chernecky R, Rossouw P, Kulkarni G. The effect of various storage methods and media on shear-bond strengths of dental composite resin to bovine dentine. archives of oral biology, 1998, 43: 305-11.

26. IA. M. Testing of dentin adhesives. Journal of dental research, 2000.

27. Robbins JW. Color characterization of porcelain veneers. Quintessence international, 1991, 22.

28. Van Meerbeek B, Perdigao J, Lambrechts $P$, Vanherle $G$. The clinical performance of adhesives. Journal of dentistry, 1998, 26: 1-20.
29. Lin T-M, Liu P-R, Ramp LC, Essig ME, Givan DA, Pan $\mathrm{Y}-\mathrm{H}$. Fracture resistance and marginal discrepancy of porcelain laminate veneers influenced by preparation design and restorative material in vitro. Journal of dentistry, 2012, 40: 202-9.

30. McLaughlin G, Morrison J. Porcelain fused to tooth-the state of the art. Restorative dentistry, 1988, 4: $90-4$.

31. Christensen GJ, Quart AM, Small CB, Klein RS, Scheitler LE, Arendt DM, Whitt CC, Hon JO, Stubenvol R. Clinical Practice. The Journal of the American Dental Association, 1991, 122: 81-93.

32. Cherukara G, Seymour K, Samarawickrama D, Zou L. A study into the variations in the labial reduction of teeth prepared to receive porcelain veneers-a comparison of three clinical techniques. British dental journal, 2002, 192: 401-4.

33. Highton R, Caputo AA. A photoelastic study of stresses on porcelain laminate preparations. The Journal of Prosthetic Dentistry, 1987, 58: 157-161.

34. Shetty A, Kaiwar A, Shubhashini N, Ashwini $P$, Naveen D, Adarsha M, Shetty M, Meena N. Survival rates of porcelain laminate restoration based on different incisal preparation designs: An analysis. Journal of conservative dentistry, 2011, 14: 10.

35. May LG, Kelly JR, Bottino MA, Hill T. Effects of cement thickness and bonding on the failure loads of CAD/CAM ceramic crowns: multi-physics FEA modeling and monotonic testing. Dental Materials, 2012, 28: e99-e109.

36. Büchi DL, Ebler S, Hämmerle $\mathrm{CH}$, Sailer I. Marginal and internal fit of curved anterior CAD/CAM-milled zirconia fixed dental prostheses: an in-vitro study. Quintessence international, 2014, 45.

37. Nakamura T, Dei N, Kojima T, Wakabayashi K. Marginal and internal fit of Cerec 3 CAD/CAM allceramic crowns. International Journal of Prosthodontics, 2003, 16.

38. Reich S, Uhlen S, Gozdowski S, Lohbauer U. Measurement of cement thickness under lithium disilicate crowns using an impression material technique. Clinical oral investigations, 2011, 15: 521-6.

39. Meijering A, Creugers N, Roeters F, Mulder J. Survival of three types of veneer restorations in a clinical trial: a 2.5-year interim evaluation. Journal of dentistry, 1998, 26: 563-8. 
40. Schmidt KK, Chiayabutr Y, Phillips KM, Kois JC. Influence of preparation design and existing condition of tooth structure on load to failure of ceramic laminate veneers. The Journal of Prosthetic Dentistry, 2011, 105: 374-82.

41. Troedson M, Dérand $T$. Effect of margin design, cement polymerization, and angle of loading on stress in porcelain veneers. The Journal of Prosthetic Dentistry, 1999, 82: 518-24.

42. Peumans $M$, De Munck J, Fieuws $S$, Lambrechts $P$, Vanherle G, Van Meerbeek B. A prospective tenyear clinical trial of porcelain veneers. Journal of Adhesive Dentistry, 2004, 6.

43. De Jager N, Pallav P, Feilzer AJ. The apparent increase of the Young's modulus in thin cement layers. Dental Materials, 2004, 20: 457-62.

44. Scherrer SS, De Rijk WG, Belser UC, Meyer J-M. Effect of cement film thickness on the fracture resistance of a machinable glass-ceramic. Dental Materials, 1994, 10: 172-7.

45. Prakki A, Cilli R, Da Costa AU, De Paiva Gonçalves SE, Mondelli L, Rafael F, Pereira JC. Effect of resin luting film thickness on fracture resistance of a ceramic cemented to dentin. Journal of Prosthodontics, 2007, 16: 172-8.

46. Burke F. Maximising the fracture resistance of dentine-bonded all-ceramic crowns. Journal of dentistry, 1999, 27: 169-73.

47. Karntiang $P$, Leevailoj C. Effect of resin cement thickness and ceramic thickness on fracture resistance of enamel-bonded ceramic. Chulalongkorn University Dental Journal, 2014, 37: 161-70.

\author{
Yazışma Adresi \\ Uzm. Dt. Yiğit Kaan POLAT \\ M.S.B Oran Lojmanları Askeri Misafirhanesi \\ Kat:4 Daire:401 \\ Çankaya/Ankara \\ Telefon: 05336198736 \\ E-posta: yigitkaanpolat@gmail.com
}

